\title{
Data Fusion Using Fuzzy Logic Techniques Supported by Modified Weighting Factors (FLMW)
}

\author{
Lukasz Fracczak $^{1} \cdot$ Leszek Podsedkowski $^{1} \cdot$ Agnieszka Kobierska $^{1}$
}

Received: 30 October 2013/Revised: 18 September 2015/Accepted: 1 October 2015/Published online: 24 October 2015

(C) The Author(s) 2015. This article is published with open access at Springerlink.com

\begin{abstract}
This article describes a data fusion algorithm that uses fuzzy logic techniques. The algorithm involves weighting factors, the values of which change depending on whether the conditions dictated by processes of fuzzy logic have been met. By modifying the values of weighting factors, one can achieve a measurement signal with expected properties. The article includes a general description of the algorithm and an example of its application. The algorithm was developed for the purposes of data fusion for contactless measurement of the linear and angular position of an automatic laparoscopic device or a laparoscopic camera (hereinafter Laparoscope sleeve or LS).

These measurements are used in the article to close the feedback loop on the position within a servomechanism with two degrees of freedom.
\end{abstract}

Keywords Fuzzy logic $\cdot$ Data fusion $\cdot$ Contactless measuring system - Two DOF measuring system . Weighting factors

\section{Introduction}

Today, numerous papers concerning data fusion are available. Publications [1-3] provide a list of such papers. The latter is a good summary and systematisation of the

Lukasz Fracczak

lukasz.fracczak@p.lodz.pl

Leszek Podsedkowski

lpodsedk@p.lodz.pl

Agnieszka Kobierska

agnieszka.kobierska@p.lodz.pl

1 Institute of Machine Tools and Production Engineering, Lodz University of Technology, Lodz, Poland applications of researchers' findings on data fusion to date. Based on the publication [2], one can conclude that algorithms are adjusted to a particular measurement issue.

The Kalman filter [4] is the most widely used algorithm in systems that measure position. Study [5] uses it to plan the correct route for commuting to a destination in an urban environment. Study [6] and Study [7] develop expanded Kalman filters. Literature also describes modified Kalman filters. For instance, Study [8] proposes a modification of the weighting matrix of a filter depending on the correlation of data estimation errors.

Interesting examples of algorithms include hybrid solutions that combine Kalman filters with other techniques. For instance, Study [9] presents the Distributed Data Fusion algorithm, which uses transmitter-receiver (TR) control. The authors of the study use the algorithm to assess and follow the position of objects within the visual field of a sensor system. Another example of a hybrid solution can be found in Studies [10, 11], in which the Kalman filter is used in conjunction with fuzzy logic techniques. The other combination of the Kalman Filter with other algorithms can be found in, e.g. state-vector with H-infinity [12] and track fusion algorithm with three fusions [13]. The authors in the paper [14] present the finite impulse response (FIR) which is similar to the Kalman Filtering but it must be used on the off-line data. However, the Kalman filters and their derivates (expanded and modified filters) cannot be used to conduct data fusion in measurement systems in which the error of one of the sensors increases with working time (for instance, in odometric assessments).

A completely different data fusion solution uses only a fuzzy logic technique with measurement weights. Study [15] describes a filter that is based on adjusting a weighting matrix to observational errors of a given sensor. In such an 
algorithm, the weight of an observational error tends to 0 when the value of the error increases (as is the case of odometric assessments or measurements using Complementary Metal-Oxide-Semiconductor (CMOS) sensor); thus, the measuring signal will not be taken into account in the output data.

Recently in a data fusion field, a significant increase of artificial intelligence (AI) algorithms used in tasks of position and velocity measurement can be noticed in a data fusion field. These algorithms are used to integrate the global positioning system (GPS) and inertial navigation system (INS) measurements [16-20]. Such systems are based on the neural networks [16-18], wavelets [18, 19] and cross-validations [16]. However, these techniques require a great number of calculations. This can indicate measurement lags, which result in instability of the servo drive. This paper presents the FLMW algorithm that can be easily computed; therefore, it can be used in the servo drive positioning loop.

The data fusion problem can be described as a function of measured data and weight coefficients. Then we can use the optimization technique to calculate the weight coefficients values adjusted to measured data. Gene expression programming (GP) suits such optimization task well for it can be used to derive more advanced function [21]. Such methods require complex computation process. Yet, the best fitting of the chromosome algorithm can be used in order to decrease this complexity [22]. The GP is used in order to derive function best fitting the setup conditions. In case where weight coefficients values change over time it becomes necessary to recalculate them which can lead to the destabilisation of the servo drive.

The low computationally data fusion model is proposed in Study [23]. This model is based on the value factors, which are proportional to the features or noise of the features of the measured object. In this method, the CMOS sensor error will be taken into account in data after fusion, which can lead to the increase of the error in time.

This article describes a data fusion algorithm that uses fuzzy logic techniques. The subsequent part of the article was divided into three chapters to facilitate describing how the presented algorithm operates. The second chapter describes an application of the data fusion algorithm, which was the object of the experimental research. In the third chapter, the general model of the algorithm was derived. Lastly, the fourth chapter summarises the conducted experiments.

\section{Measurements System}

A measurement system was developed and tested for a servo drive designed for the RobIn Heart cardiac surgery robot and it is described as follows. [24-31]. In the future, this servo drive [31] will replace the traditional linear-telescopic drive that moves the LS along its axis. Replacing the traditional drive will expand functionality (by adding a degree of freedom) and allow reduction the size of the cardiac surgery robot.

The servo drive uses a friction connection between the drive mechanism and the laparoscope bushing. This approach to transferring drive requires measuring the position of the LS. Such a measurement is complicated; it takes place within two degrees of freedom and due to medical regulations, it needs to be contactless. That is why it is necessary to use two independent measurement subsystems and an appropriate data fusion algorithm.

\subsection{Laser Triangulation Rangefinders}

One of the subsystems is based on laser triangulation rangefinders (TR) (Fig. 1) and includes two sensors. Thus, one TR measures the distance between the base of the spiral and the drive mechanism (linear position), while the second TR measures the position of the spiral surface of the measuring element. The angular position of the bushing in relation to the drive mechanism is given by the equation coming directly from the Fig. 1:

$a_{\phi T}=\frac{\left(l_{1}-l_{2}\right)}{r_{s} \tan \alpha_{s}}$

while the linear position is given by the equation:

$a_{L T}=l_{1}$

where $a_{\phi T}$ and $a_{L T}$ are the angular an linear position based on the TR, respectively, $r_{s}$ is radius of the measurement spiral, $l_{1}$ and $l_{2}$ are the values obtained by the TR aimed at the base and the spiral surface of the measuring element, respectively, and $\alpha_{s}$ is the elevation angle of the spiral surface.

The advantage of this measurement system is its high repeatability. On the other hand, its disadvantages are low

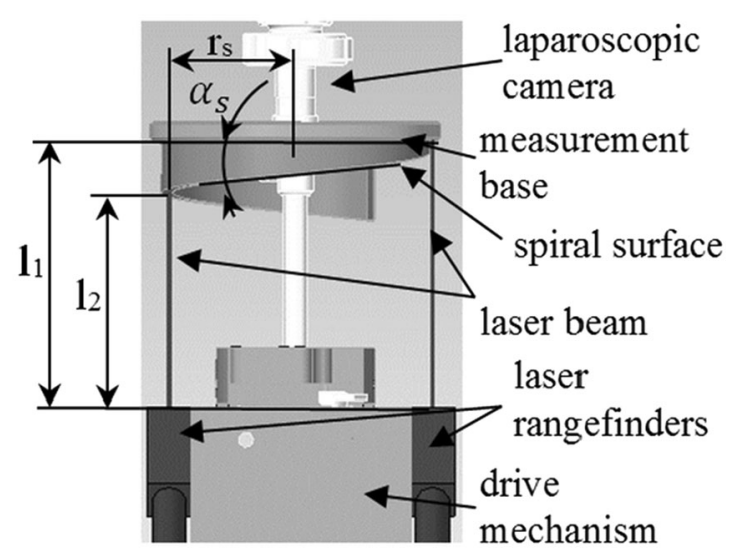

Fig. 1 Measurement using laser triangulation rangefinders 
measurement resolution, noise in the measurement signal and discontinuity point of the spiral that introduces measurement insensitivity ranging about $5^{\circ}$ (this error can be eliminated by introducing appropriate programme limits into the automatic control system).

\subsection{Complementary Metal-Oxide-Semiconductor (CMOS) Sensor}

CMOS sensors operate based on a comparison between two images taken of a front surface with a small CMOS camera. The sensor includes a system that follows the movement of individual points of the surface in subsequent images and transmits a signal concerning the displacement to an integrated circuit that works with a computer. The resolution achievable by these sensors is given by the dot per inch (DPI) value, included in the documentation. For instance, for a CMOS sensor with 1000 DPI, measurement resolution equals $0.0244 \mathrm{~mm}$, which is high compared to the resolution of a triangulation rangefinder. The problem with using these sensors is their low accuracy and measurement repeatability as well as their high sensitivity to changes of the measurement surface.

Because CMOS sensors measure displacement while TR measure position, the measurement value of CMOS sensors is adjusted to measurement of position. To achieve this, the value obtained by the TR (average value from 50 measurements with inactive drive, taken immediately after activating the measurement system) is entered as the first measurement. During subsequent steps of the iteration, the value of gain measured by the CMOS is added to the base value according to the formula:

$a_{L C(k)}=\left\{\begin{array}{lll}a_{\text {baze }} & \text { for } & k=0 \\ l_{L 2(k-1)}+\Delta a_{L C(k)} & \text { for } & k \geq 1\end{array}\right.$

where $\Delta a_{L C(k)}$ is the displacement detected by the CMOS sensor, $a_{L C(k)}$ is the measurement signal of the CMOS sensor in the linear position in the current step of the iteration, $l_{L 2(k-1)}$ is the value of the measurement signal obtained by the data fusion algorithm in the previous step of the iteration, and $a_{\text {baze }}$ is the base value obtained by the TR.

In this study, subscript $k$ refers to the value in the $k$-th step of the iteration, while $k-1$ refers to the value in the $k-1$-th preceding step.

The value of the angular measurement $\left(a_{\phi C(k)}\right)$ is caclulated in an analogous manner.

\subsection{Data Fusion Algorithm}

Data fusion takes place separately for the linear direction and the angular direction. Because the algorithm operates analogously in both directions of measurement, only equations for the linear direction will be derived in this article. The complete model of the data fusion algorithm will be presented in the final matrix equation and will include both measurement directions.

Measurement systems were selected in a way that enabled a vast range of properties: triangulation rangefinders have high accuracy and repeatability of measurement, while CMOS sensors have high resolution and low accuracy. Such a selection of sensors makes it problematic to conduct measurements with both high resolution and high accuracy. To obtain such a measurement, a special data fusion algorithm was developed on the basis of elements of fuzzy logic. In the first stage of data fusion, the value of the higher resolution measurement is expected to lie within the observational error of the high-accuracy sensor. For this reason, the following logical condition is created:

$A_{L 1}:=\left\{\left|a_{L C(k)}-a_{L T(k)}\right|<\sigma a_{L T}\right\}$

where $A_{L 1}$ is the logical condition, and $\sigma a_{L T}$ is the measuring error of the triangulation rangefinder.

Next, products of weights and of measurement values are introduced.

$l_{L 1(k)}=a_{L C(k)} * w_{L 11(k)}+a_{L T(k)} * w_{L 12(k)}$

where $l_{L 1(k)}$ is the value of the measurement after data fusion, and $w_{L 11(k)}, w_{L 12(k)}$ are values of weights of individual measurement signals.

Then, depending on whether the $A_{L 1}$ condition was met, the significance of measured values is changed into the final post-fusion value. The significance is changed by modifying the values of weights in a given step of the iteration. Each change is described by the relationship below:

$$
\begin{array}{r}
A_{L 1}=\text { true }=>\left\{\begin{array}{l}
w_{L 11(k)}=w_{L 11(k-1)}+0.02 \\
w_{L 12(k)}=w_{L 12(k-1)}-0.02
\end{array}\right. \\
A_{L 1}=\text { false }=>\left\{\begin{array}{l}
w_{L 11(k)}=w_{L 11(k-1)}-0.02 \\
w_{L 12(k)}=w_{L 12(k-1)}+0.02
\end{array}\right.
\end{array}
$$

with the following boundary conditions:

$$
\begin{aligned}
& 0.1 \leq w_{L 11(k)} \leq 0.9 \\
& 0.1 \leq w_{L 12(k)} \leq 0.9 \\
& w_{L 11(k)}+w_{L 12(k)}=1
\end{aligned}
$$

Thanks to this algorithm, the measurement value after data fusion will lay within the observational error of the highaccuracy sensors.

Research has shown that when the servo drive was in motion, a considerable disturbance of the measure appeared due to the uneven surface of the measurement 
spiral. For this reason, a second condition was added to the algorithm. The condition is based on the assumption that when the servo drive is in motion, the measurement signal from the CMOS sensor is more significant (it is not affected by the uneven surface of the spiral). This condition can be applied because high measurement accuracy is desirable during low-speed motion and in the final stage of the motion, i.e. positioning. That is why a speed condition can be introduced to the algorithm: when the moving servo drive exceeds a certain speed value, the data fusion algorithm will increase the significance of measurement values from the CMOS sensor. On the other hand, at a low speed, the algorithm will increase the weight of measurement values from the previous part of the data fusion algorithm. The mathematical description of the condition is as follows:

$$
\begin{aligned}
& A_{L 2}:=\left\{\left|\frac{\Delta a_{L C(k)}}{\Delta t_{L}}\right|<V_{L g}\right\} \\
& A_{L 2}=\text { true }=>\left\{\begin{array}{l}
w_{L 21(k)}=w_{L 21(k-1)}+0.01 \\
w_{L 22(k)}=w_{L 22(k-1)}-0.01
\end{array}\right. \\
& A_{L 2}=\text { false }=>\left\{\begin{array}{l}
w_{L 21(k)}=w_{L 21(k-1)}-0.01 \\
w_{L 22(k)}=w_{L 22(k-1)}+0.01
\end{array}\right.
\end{aligned}
$$

The data fusion equation is introduced:

$$
l_{L 2(k)}=a_{L C(k)} * w_{L 21(k)}+l_{L 1(k)} * w_{L 22(k)}
$$

with the following boundary conditions:

$$
\begin{aligned}
& 0.1 \leq w_{L 21(k)} \leq 0.9 \\
& 0.1 \leq w_{L 22(k)} \leq 0.9 \\
& w_{L 21(k)}+w_{L 22(k)}=1
\end{aligned}
$$

where $A_{L 2}$ is the second logical condition $\Delta t_{L}$ is the duration of a given step of the iteration, $V_{L g}$ is the threshold value (if it is exceeded, the significance of the CMOS signal increases), and $w_{L 21(k)}, w_{L 22(k)}$ are weights of measurement signals from the CMOS and post-fusion values from the first part of the algorithm, respectively, and $l_{L 2(k)}$ is the value of the measurement after the second step data fusion.

Ultimately, the entire data fusion algorithm for one measurement direction can be expressed in the form of two matrix equations:

$$
\begin{aligned}
& l_{L 1(k)}=\left[\begin{array}{ll}
w_{L 11(k)} & w_{L 12(k)}
\end{array}\right]\left[\begin{array}{c}
a_{L C(k)} \\
a_{L T(k)}
\end{array}\right] \\
& l_{L 2(k)}=\left[\begin{array}{lll}
w_{L 21(k)} & 0 & w_{L 22(k)}
\end{array}\right]\left[\begin{array}{c}
a_{L C(k)} \\
a_{L T(k)} \\
l_{L 1(k)}
\end{array}\right]
\end{aligned}
$$

Conversely, the system of equations for both directions is as follows:

$$
\begin{aligned}
& L_{1}=W_{1} A_{1} \\
& L_{2}=W_{2} A_{2}
\end{aligned}
$$

where the matrixes are equal:

$$
\begin{aligned}
& L_{1}=\left[\begin{array}{c}
l_{L 1(k)} \\
l_{\phi 1(k)}
\end{array}\right] \quad L_{2}=\left[\begin{array}{l}
l_{L 2(k)} \\
l_{\phi 2(k)}
\end{array}\right] \\
& W_{1}=\left[\begin{array}{cccc}
w_{L 11(k)} & w_{L 12(k)} & 0 & 0 \\
0 & 0 & w_{\phi 11(k)} & w_{\phi 12(k)}
\end{array}\right] \\
& W_{2}=\left[\begin{array}{cccccc}
w_{L 21(k)} & 0 & 0 & 0 & w_{L 22(k)} & 0 \\
0 & 0 & w_{\phi 21(k)} & 0 & 0 & w_{\phi 22(k)}
\end{array}\right] \\
& A_{1}=\left[\begin{array}{llll}
a_{L C(k)} & a_{L T(k)} & a_{\phi C(k)} & a_{\phi T(k)}
\end{array}\right]^{T} \\
& A_{2}=\left[\begin{array}{llllll}
a_{L C(k)} & a_{L T(k)} & a_{\phi C(k)} & a_{\phi T(k)} & l_{L 1(k)} & l_{\phi 1(k)}
\end{array}\right]^{T}
\end{aligned}
$$

where $l_{L 1(k)}, l_{\phi 1(k)}$ are output signals obtained in the first part of the data fusion algorithm in the linear and angular direction, respectively, $l_{L 2(k)}, l_{\phi 2(k)}$ are output signals from the second part of the data fusion algorithm in the linear and angular direction, respectively, and $w_{L i j(k)}, w_{\phi i j(k)}$ are the weights of the $j$-th measurement signal in the $k$-th step of the iteration from the $i$-th part of the algorithm for the linear and angular direction, respectively.

The two equations above were edited in a way that would allow us to present the iterative application of weighting equations and to show the method of introducing subsequent equations that include, for instance, the relationships between the linear and angular measurements (due to, for example, an oblique mounting of the CMOS sensor). After substituting Eq. (8) into Eq. (9), the final equation takes the following form:

$$
\left[\begin{array}{l}
l_{L(k)} \\
l_{\phi(k)}
\end{array}\right]=\left[\begin{array}{cccc}
a_{11} & a_{12} & 0 & 0 \\
0 & 0 & a_{23} & a_{24}
\end{array}\right]\left[\begin{array}{l}
a_{L C(k)} \\
a_{L T(k)} \\
a_{\phi C(k)} \\
a_{\phi T(k)}
\end{array}\right]
$$

where:

$$
\begin{aligned}
& a_{11}=w_{L 21(k)}+w_{L 22(k)} w_{L 11(k)} \\
& a_{12}=w_{L 22(k)} w_{L 12(k)} \\
& a_{23}=w_{\phi 21(k)}+w_{\phi 22(k)} w_{\phi 11(k)} \\
& a_{24}=w_{\phi 22(k)} w_{\phi 12(k)} .
\end{aligned}
$$

\subsection{Tests of the Measurement System}

Figure 2 shows the test stand in which the data fusion algorithm was tested. The test stand consisted of a servo 


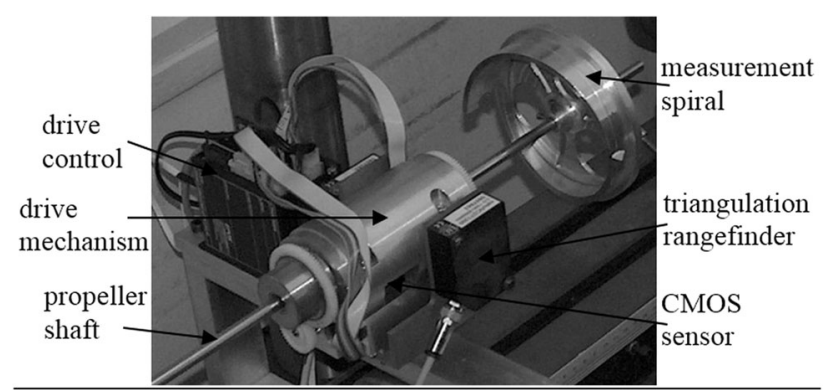

Fig. 2 The test stand

drive that forced a propeller shaft (LS) to move in two directions.

A CMOS sensor was attached below the propeller shaft. It takes measurements on the circumferential surface of the LS in directions: linear and angular (parallel and perpendicular to the axis of the shaft, respectively). The TR are mounted next to the body of the servo drive and measure the position of the spiral mounted on the LS in relation to the body of the servo drive. This test stand was used to test uncertainty measurement and resolution separately for both directions of movement: linear and angular. The measurement uncertainty test was performed for linear and angular motion separately. It has been repeated 50 times for LS $10 \mathrm{~mm}$ linear displacement starting from one point and moving towards specified direction. The test was repeated with 5 different starting points. For angular direction measurement, a $50^{\circ}$ displacement has been used. The uncertainty was determined with $0.05 \%$ confidence level. The resolution tests were based on the slight displacements of the LS. It has shown when the measurement took place and what was its value. The induction sensors with $0.01 \mathrm{~mm}$ accuracy were used for the reference measurement. Table 1 shows the results of the test.

The results of the test allow us to conclude that the introduction of the data fusion algorithm improved measurement accuracy and resolution.

The next test involved moving the LS into a given position and then returning it to the initial position. Because the results were similar for both the linear and the angular directions, this article only presents the test for the linear direction (Fig. 3). As a reference algorithm, the Kalman filter was used, described by the following equations (symbols used are taken from Publication [9]): prediction

$X_{k}^{-}=A X_{k-1}$

$P_{k}^{-}=A P_{k-1}^{-} A^{T}+Q$

corection

$K_{k}=\frac{P_{k}^{-} H^{T}}{H P_{k}^{-} H^{T}+R}$
Table 1 Result of the accuracy and resolution test

\begin{tabular}{llll}
\hline & TR & CMOS & FLMW \\
\hline Linear direction (mm) & & & \\
Accuracy & \pm 0.4 & \pm 0.6 & \pm 0.1 \\
Resolution & 0.1 & 0.02 & 0.01 \\
Angular direction $\left({ }^{\circ}\right)$ & & & \\
Accuracy & \pm 8.8 & \pm 15.4 & \pm 0.4 \\
Resolution & 2.2 & 0.6 & 0.1 \\
\hline
\end{tabular}

$X_{k}=X_{k}^{-}+K_{k}\left(Z_{k}-H X_{k}^{-}\right)$

with individual matrixes equal to:

$A=\left[\begin{array}{cccc}1 & 0 & \Delta t & 0 \\ 0 & 1 & 0 & \Delta t \\ 0 & 0 & 1 & 0 \\ 0 & 0 & 0 & 1\end{array}\right] \quad Q=\left[\begin{array}{cccc}0.01 & 0 & 0 & 0 \\ 0 & 0.01 & 0 & 0 \\ 0 & 0 & 1 & 0 \\ 0 & 0 & 0 & 1\end{array}\right]$

$H=\left[\begin{array}{llll}1 & 0 & 0 & 0 \\ 0 & 1 & 0 & 0\end{array}\right] \quad R=\left[\begin{array}{cc}0.1 & 0 \\ 0 & 0.1\end{array}\right]$

$Z_{k}=\left[\begin{array}{c}a_{L T} * 0.9+z_{L(k)} * 0.1 \\ a_{\phi T} * 0.9+z_{\phi(k)} * 0.1\end{array}\right]$

where:

$z_{L(k)}= \begin{cases}a_{\text {baze }} & \text { for } \quad k=0 \\ z_{L(k-1)}+\Delta a_{L C(k)} & \text { for } \quad k \geq 1\end{cases}$

The $z_{\phi(k)}$ is caclulated in an analogous manner.

As Fig. 3 shows, the FLMW algorithm reduces noise in the measurement signal much better than the Kalman Filter algorithm. The observational error of the CMOS sensor does not translate into the post-fusion measurement. Thus, the signal follows measurements performed by sensors with much greater measurement repeatability. The postfusion signal with the FLMW algorithm applied constantly reduces noise while steadily following the signals from the measurement sensors.

The next test assessed the effect of the algorithm on the damping and delaying of the measurement signal. After the test, the amplitude-frequency response diagram (Fig. 5) and the phase diagram (Fig. 6) was created for both directions (linear and angular) in which the measurement system moved. Figure 4 shows an example response signal during input harmonic function in the linear direction.

Amplitude-frequency response diagrams were created using the relationship: $20 \log \left(\frac{A_{y}}{A_{x}}\right)$ where: $A_{x}$ is the input amplitude (the signal from the TR sensor) and $A_{y}$ is the value after data fusion. 
(a)

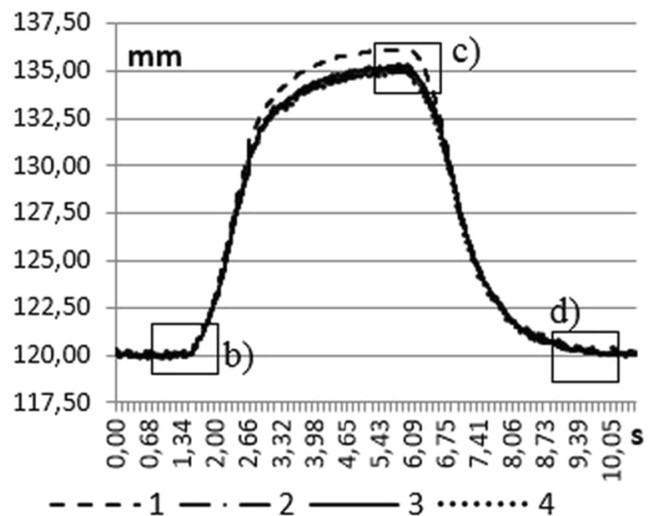

(c)

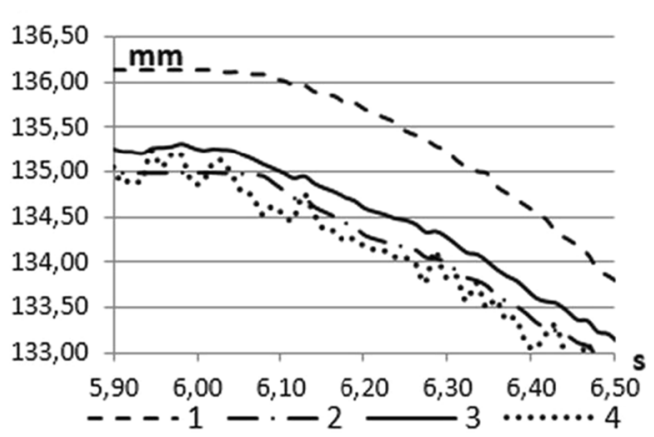

(b)

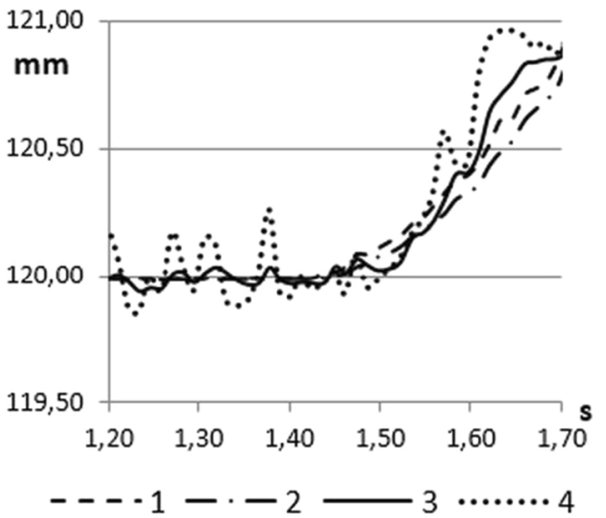

(d)

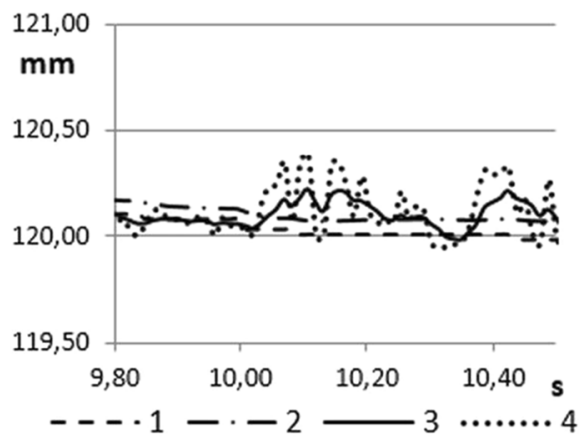

Fig. 3 Test of the response of algorithms to linear displacement: a the entire test, b-d elements of the test where $1 z_{L}, 2$ the FLMW algorithm, 3 the Kalman Filter, 4 the TR sensor

Amplitude-frequency response diagrams show that the measurement signal is dampened during harmonic excitation of more than $1 \mathrm{~Hz}$. On the other hand, phasic characteristics show that the system does not display increased delay for the angular direction. We can conclude that with the aforementioned fuzzy logic conditions and parameters of changes in the weighting factors, the algorithm should work in measurement systems with harmonic excitation of less than $1 \mathrm{~Hz}$.

\section{General Model of the Data Fusion Algorithm}

In order to derive the general mathematical model, we assume that the measurement system consists of $n$ independent sensors that emit signals $a_{1}, \ldots, a_{n}$. These sensors are used to determine the state of $q$ independent variables marked $l_{1}, \ldots, l_{q}$. Next, a prediction is made concerning which of the measurement signals contributes most to exposing the expected post-fusion measurement property. In the following step, logical conditions are created that will unambiguously indicate the sensor (or sensors) most significant from the point of view of the given measurement property. Next, a $Q$ set of logical conditions is created.

$\forall A_{j} \in Q$

$A_{j}=f\left(a_{1}, a_{2}, \ldots, a_{n}\right)$

where: $A_{j}$ is the $j$-th logical condition and $a_{i}$ is the measurement value from the $n$-th sensor.

For each logical condition, the post-fusion measurement is given by the sum of products of weight and values of measurement signals, as described below:

$$
\begin{aligned}
& l_{A j(k)}=a_{1(k)} w_{A j 1(k)}+\ldots+a_{n(k)} w_{A j n(k)} \\
& l_{A j(k)}=\sum_{i=1}^{n} a_{i(k)} w_{A j i(k)}
\end{aligned}
$$

where $l_{A j(k)}$ is the $j$-th post-fusion variable measured after testing the $j$-th logical condition and $a_{i(k)}$ is the $i$-th measurement signal, $w_{A j i(k)}$ is the weight of the $j$-th condition of the $i$-th measurement signal.

Next, if a given logical condition is met, changes in the weights of measurement signals are predicted (if the condition is not met, then an opposite change in weight is predicted). 


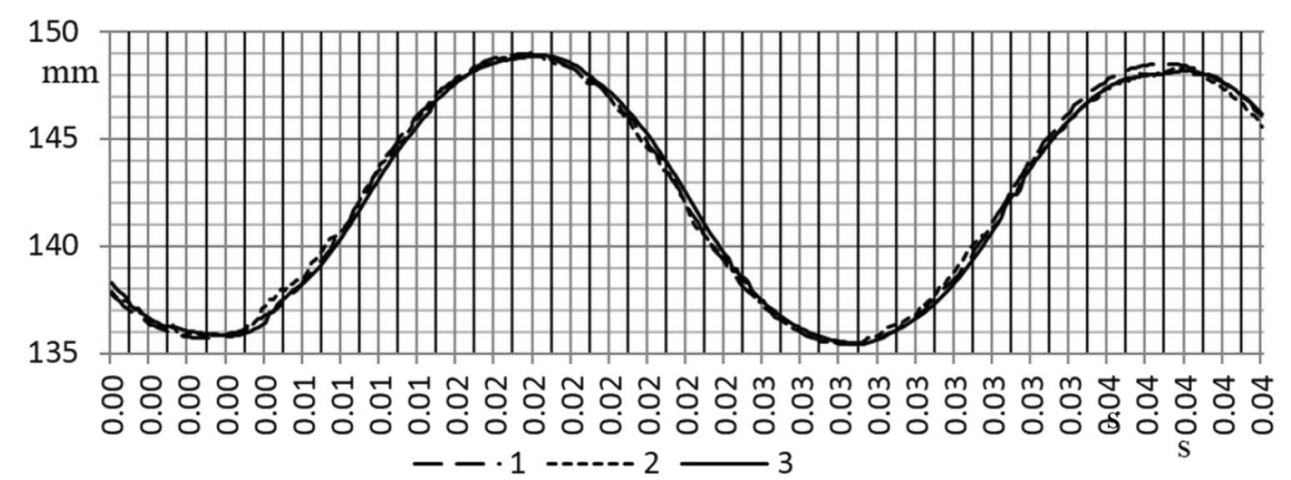

Fig. 4 Response of the measurement system to harmonic excitation at frequency of $0.4 \mathrm{~Hz}$ and with amplitude of $13 \mathrm{~mm}$ where 1 the reference value, 2 the TR sensor, 3 the FLMW algorithm

(a)

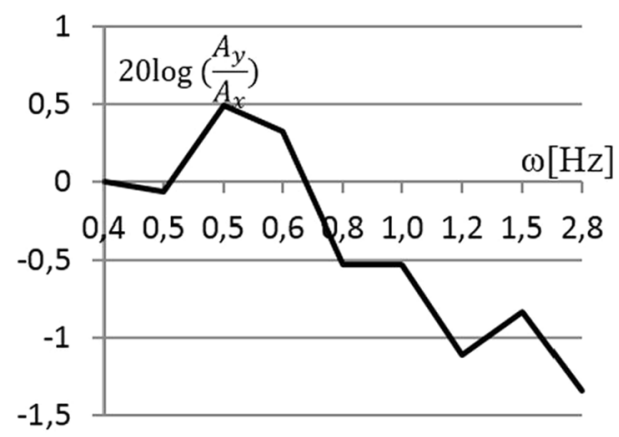

(b)

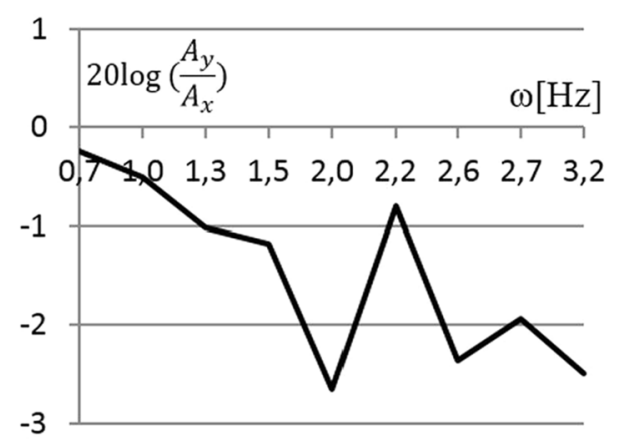

Fig. 5 Amplitude-frequency response diagrams of the signal after data fusion: a linear direction, $\mathbf{b}$ angular direction

(a)

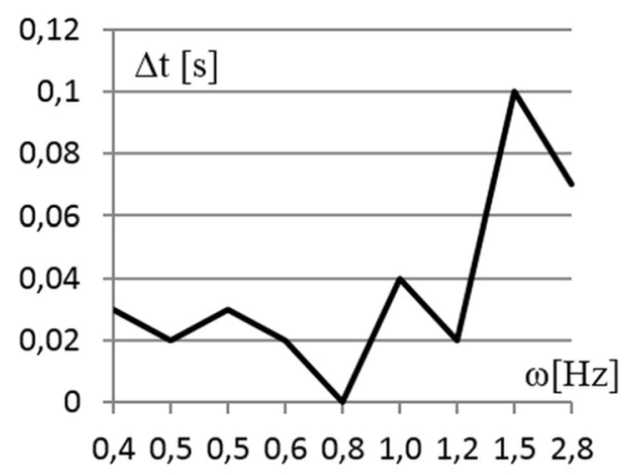

(b)

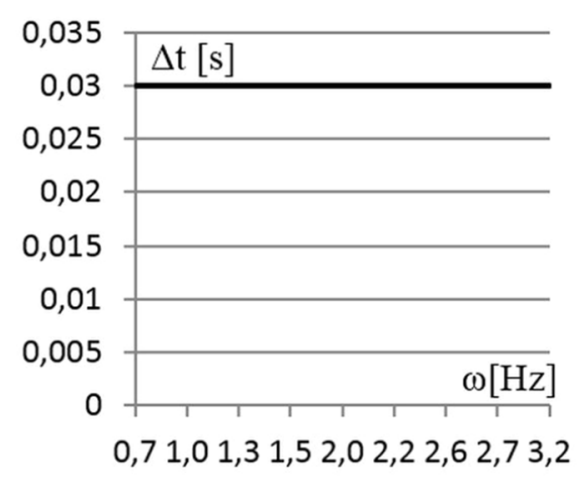

Fig. 6 Phase diagrams of the signal after data fusion: a linear direction, b angular direction

$$
\begin{array}{cc}
A_{j}=\text { true }=> & w_{A j i(k)}=w_{A j i(k-1)}+\Delta w_{A j i} \\
A_{j}=\text { false }=> & w_{A j i(k)}=w_{A j i(k-1)}-\Delta w_{A j i}
\end{array}
$$

where $i=1,2, \ldots, n$ and $j=1,2, \ldots, q$

with the following boundary conditions:

$$
\begin{gathered}
0<w_{A j i(k)}<1 \\
\sum_{i=1}^{n} w_{A j i(k)}=1
\end{gathered}
$$

where $w_{A j i(k)}, w_{A j i(k-1)}$ are the values of the $i$-th measurement signal for the $j$-th logical condition in the $k$-th and $(k-1)$-th measurement step, and $\Delta w_{A j i}$ is the value by which a given weighting factor changes.

The following matrix equation of data fusion can be constructed based on the above considerations:

$L_{A}=W_{A} A$ 
where each matrix in a given data fusion step is as follows:

$L_{A}=\left[\begin{array}{c}l_{A 1} \\ \vdots \\ l_{A q}\end{array}\right] W_{A}=\left[\begin{array}{ccc}w_{A 11(k)} & \ldots & w_{A 1 n(k)} \\ \vdots & \vdots & \vdots \\ w_{A q 1(k)} & \ldots & w_{A q n(k)}\end{array}\right]$

$A=\left[\begin{array}{lll}a_{1} & \ldots & a_{n}\end{array}\right]^{T}$

where $L_{A}$ is the matrix of measurement signals after data fusion, $W_{A}$ is the matrix of weights, and $A$ is the matrix of signals from measurement sensors.

If the post-fusion signal needs to be introduced into another fusion equation, then $r$ new logical conditions are created as follows:

$$
\begin{aligned}
& \forall B_{t} \in Q \\
& B_{t}=f\left(a_{1}, a_{2}, \ldots, a_{n}, l_{1}, \ldots, l_{q}\right) \quad \text { where } t=1,2, \ldots, r
\end{aligned}
$$

where $B_{t}$ is the $t$-th logical condition and $a_{i}$ is the measurement value from the $n$-th sensor, and $l_{i}$ is the value after the $i$-th logical condition.

The remaining part of the procedure is analogous to the first part of the algorithm. The final matrix equation in the second part is created by an adequate expansion of the matrix. Thus, we can state that:

$L_{B}=W_{B} B$

$L_{B}=\left[\begin{array}{lll}l_{B 1} & \ldots & l_{B r}\end{array}\right]^{T}$

$B=\left[\begin{array}{lllll}a_{1} & \ldots & a_{n} & l_{A 1} & l_{A q}\end{array}\right]^{T}$

$W_{B}=\left[\begin{array}{cccccc}w_{B 11(k)} & \ldots & w_{B 1 n(k)} & w_{B 1(n+1)(k)} & \ldots & w_{B 1 m(k)} \\ \vdots & \vdots & \vdots & \vdots & \vdots & \vdots \\ w_{B r 1(k)} & \ldots & w_{B r n(k)} & w_{B r(n+1)(k)} & \ldots & w_{B r m(k)}\end{array}\right]$

where $m=n+q$ with the following boundary conditions:

$$
\begin{aligned}
0 \leq w_{B t s} & \leq 1 \\
\sum_{s=1}^{m} w_{B t s(k)} & =1
\end{aligned}
$$

where $s=1,2, \ldots, m$

where $L_{B}$ is the matrix of measurement signals after data fusion, $W_{B}$ is the matrix of weights in the B step, and $B$ is the matrix of signals from measurement sensors and data after fusion from the previous step.

As a result of this algorithm, the $l_{B r}$ measurement value is the output signal from the data fusion algorithm. However, each of the $l_{A j}$ or $l_{B t}$ signals can constitute an individual output signal with particular properties. By using the procedure described above, additional parts of the data fusion algorithm can be introduced to achieve expected measurement properties.

\section{Summary}

This article presents a general model of a data fusion algorithm based on the system of fuzzy logic and supported by modified weighting factors. The algorithm was implemented into a contactless measurement system that consisted of a servo drive with the drive transmitted by friction in two degrees of freedom. The use of such an algorithm allowed us to obtain a measurement with particular properties (high accuracy and repeatability of positioning and high resolution). The crucial part of the algorithm is a new approach to data fusion: it makes use of the properties of each measurement sensor without combining them through a complicated mathematical model. Moreover, the algorithm sets no limits on the number of sensors used and, if many conditions of data fusion are introduced, it is able to operate based on previously determined values. A major advantage of the algorithm is the possibility that all these values can be used in every part of the algorithm.

Acknowledgments This scientific study was financed from research funds for the years 2011-2014 as research Project No. NCN 2011/01/ B/ST7/04011 and N N502 339236.

Open Access This article is distributed under the terms of the Creative Commons Attribution 4.0 International License (http://crea tivecommons.org/licenses/by/4.0/), which permits unrestricted use, distribution, and reproduction in any medium, provided you give appropriate credit to the original author(s) and the source, provide a link to the Creative Commons license, and indicate if changes were made.

\section{References}

1. Kokar, M., Kim, K.: Review of Multisensor Data Fusion Architectures and Techniques. In: Proceedings of the 1993 International Symposium on Intelligent Control, Chicago (1993)

2. Khaleghi, B., Khamis, A., Karray, F.O. Multisensor data fusion: A review of the state-of-the-art. Inf. Fusion 14, 1-17 (2011)

3. Esteban, J., Starr, A., Willetts, R., Hannah, P., Bryanston-Cross, P.: A review of data fusion models and architectures towards engineering guidelines. Neural Comput. Appl. 14(4), 273-281 (2005)

4. Welch, G., Bishop, G. An Introduction to the Kalman Filter. Department of Computer Science. University of North Carolinam Technical Report TR-95-041 (1995)

5. Sun, S.I.: Multi-sensor optimal information fusion Kalman filters with applications. Aerosp. Sci. Technol. 8(1), 57-62 (2003)

6. Hoshino, M., Gunji, Y., Oho, S.: A Kalman Filter to Estimate Direction for Automotive Navigation. In: Proceedings of the 1996 IEEE/SICE/ RSJ International Conference on Multisensor Fusion and Integration for Inteligent Systems (1996)

7. Perala, T., Piche, R.: Robust Extended Kalman Filtering in Hybrid Positioning Applications. In: 4th Workshop on Positioning, Navigation and Communication, (WPNC'07) Hannower Germany (2007)

8. Yuan, K., Wang, H., Zhang, H.: Robot Position Realization Based on Mutli-sensor Information Fusion Algorithm. In: Fourth International Symposium on Computational Intelligence and Design (2011) 
9. Matsuzak,i T., Kameda, H.: Distributed Data Fusion with data T/R Control for Target Tracking. SICE Annual Conference (2012)

10. Al-Dhaher A.H.G., Mackesy, D.: Muti-Sensor Data Fusion Architecture Haptic, Audio and Visual Environments and Their Applications, HAVE 2004. In: Proceedings the 3rd IEEE International Workshop on (2004)

11. Li J., Lei Y., Cai Y., He L.: Multi-Sensor Data Fusion Algorithm Based on Fuzzy Adaptive Kalman Filter. In: 32nd Chinese Control Conference (CCC) (2013)

12. Meti, S.A., Sangam, V.G.: Biological Sensor Performance Validation Using Fusion Technique, Industrial Instrumentation and Control (ICIC), International Conference on, pp.1158-1163 (2015)

13. Shi J., Zhou Y., Zhang H., Zhang T., Wang L., Ren W., Luan M., Liu H.: A Multi-MEMS Sensors Information Fusion Algorithm. In: The 26th Chinese Control and Decision Conference (2014)

14. Podsedkowski, L., Podsedkowska, H.: Off-line Estimation of Trajectory in Discrete State Space Using the Minimal-Covariance Adaptive FIR Smoothing with Extended Output Vector, Methods and Models in Automation and Robotics (MMAR). In: 9th International Conference on (in press) (2015)

15. Xiao-wei, L., et al.: Multi-Sensor Weighted Fusion Algorithm Based on Fuzzy Supported Degree. Computer Science Information Technology. In: 3rd IEEE International Conference on (2010)

16. Noureldin, A., El-Shae, A., Taha, M.R.: Optimizing neuro-fuzzy modules for data fusion of vehicular navigation systems using temporal cross-validation Eng. Appl. Artif. Intell. 20(1), 49-61 (2007)

17. Noureldin, A., El-Shae, A., Bayoumi, M.: GPS/INS integration utilizing dynamic neural networks for vehicular navigation. Inf. Fusion 12(1), 48-57 (2011)

18. Shen, X.C.C., Zhang, W., Tomizuka, M., Xu, Y., Chiu, K.: Novel hybrid of strong tracking Kalman filter and wavelet neural network for GPS/INS during GPS outages. Measurement 46(10), 3847-3854 (2013)

19. Zhang, T., Xu, X.: A new method of seamless land navigation for GPS/INS integrated system. Measurement 45(4), 691-701 (2012)

20. Nassar, S., Noureldin, A., El-Sheimy, N.: Improving positioning accuracy during kinematic DGPS outage periods using SINS/ DGPS integration and SINS data de-noising. Surv. Rev. 37(292), 426-438 (2004)

21. Gandomi, A.H., Alavi, A.H., Arjmandi, P., et al.: Genetic programming and orthogonal least squares: a hybrid approach to modeling the compressive strength of CFRP-conned concrete cylinders. J. Mech. Mater. Struct. 5, 735-753 (2010)

22. Li, H., Jiao, Y.C., Zhang, L., Gu, Z.W.: Genetic algorithm based on the orthogonal design for multidimensional knapsack problems. In: Jiao L. (eds.) Adv. Nat. Comput. Lecture Notes in Computer Science, vol. 4221, pp. 696-705. Springer, New York (2006)

23. Quadri, S.A., Sidek, O.: Multisensor data fusion algorithm using factor analysis method. Int. J. Adv. Sci. Technol. 55, 43-52 (2013)

24. Podsedkowski, L., Zak, P.: Tests on cardiosurgical robot robin heart 3. Lect. Notes Control Inf. Sci. 396, 433-442 (2009)
25. Nawrat, Z., Podsedkowski, L. et al.: RobIn Heart in 2002-Actual State of Polish Cardio-Robot Robot Motion and Control. RoMoCo '02. In: Proceedings of the Third International Workshop on, pp. 33-38 (2002)

26. Nawrat, Z., Kostka, P., et al.: The advances of Robin heart Uni system-from virtual to real model, from laboratory to first vivo experiments. Int. J. Artif. Organs 32(7), 433-433 (2009)

27. Fracczak, L., Podsedkowski, L., Moll, M.: The Average 6 DOF Path Estimation with the use of Initial Approximation Curve, Methods and Models in Automation and Robotics (MMAR). In: 19th International Conference on, pp. 212-215 (2014)

28. Kobierska, A.: Force sensor for laparoscopic tool of RobIn Heart robot. Solid State Phenom. 199, 309-314 (2013)

29. Zak, P.: Using 9-axis sensor for precise cardiosurgical robot master angular position determination. Solid State Phenom. 199, 356-361 (2013)

30. Podsedkowski, L.R., Moll, J., Moll, M., Fraacczak, L.: Are the surgeon's movements repeatable? An analysis of the feasibility and expediency of implementing support procedures guiding the surgical tools and increasing motion accuracy during the performance of stereotypical movements by the surgeon. Kardiochirurgia i Torakochirurgia Polska 11(1), 90-101 (2014)

31. Fracczak, L.: Mathematical model of the servo drive with friction wheals. Simulations and real object examination results. Solid State Phenom. 198, 15-20 (2013)

Lukasz Fracczak received his Ph.D. degree in 2011 at the Faculty of Mechanical Engineering, Lodz University of Technology, Poland. His research interest includes Medical Engineering, e.g. surgical robots and gastrointestinal tract diagnosis robots and capsules. One of the latest researches is a data fusion for the contactless measuring systems for the medical devices. He is an author of several publications in these fields as well as presentations at national and international conferences.

Leszek Podsedkowski received all his scientific degrees at Lodz University of Technology, Faculty of Mechanical Engineering: M.Sc. in 1989, Ph.D. in 1993, Habilitation in 1999 and professorship in 2012. One of his research fields is medical robotics. He successfully accomplished creation of one of the most innovative devices used by surgeons-a cardiosurgical robot RobIn Heart, which has many innovative solutions: a telescopic structure of linear drive, a system of changeable tools, force sensor and force feedback. Another field of his research is planning of motion trajectories for nonholonomic mobile robots in varying work field. Creation of new $\mathrm{L}^{*}$ algorithm for scanning graphs with $\mathrm{O}(\mathrm{n})$ computational complexity is his latest achievement.

Agnieszka Kobierska received his Ph.D. degree in 2010 at the Faculty of Mechanical Engineering, Lodz University of Technology, Poland. Her research interest includes Medical Engineering, e.g. surgical robots with particular emphasis on problems related to measurement of forces. She is the author of several publications in these fields as well as presentations at national and international conferences. 